\title{
Design of Low Voltage Low Noise Amplifier for 800MHz WSN Applications*
}

\author{
ZhaolongWu, ZhiqunLi ${ }^{+}$ \\ Institute of RF- \& OE-ICs, Southeast University, Nanjing, 210096; \\ School of Integrated Circuits, Southeast University, Nanjing, 210096; \\ Key Laboratory of Jiangsu Province Sensor Network Technology, Wuxi, 214135 \\ ${ }^{+}$Corresponding author: zhiqunli@seu.edu.cn
}

\begin{abstract}
This paper presents a 1V low noise amplifier (LNA) for 700 900MHz WSN applications in $0.18 \mu \mathrm{m}$ RF CMOS process.The LNA uses capacitor cross-coupled, current-reused and noise cancellation techniques to reduce noise and power consumption. The simulation results are as follows: The voltage gain is variable with high gain at $22 \mathrm{~dB}$ and low gain at $0 \mathrm{~dB}$. At low gain mode, the input referenced $1 \mathrm{~dB}$ compression point (IP1dB) is $-15 \mathrm{dBm}$ and the input 3rd intercept point (IIP3) is $1 \mathrm{dBm}$. The noise figure (NF) at high gain is $2.85 \mathrm{~dB}$ (@800MHz).It consumes about $0.9 \mathrm{~mA}$ current from $1 \mathrm{~V}$ power supply.
\end{abstract}

Key words:low noise amplifier; low voltage;low power consumption; capacitor cross-coupled; current reuse;

Recently, research of wireless sensor network(WSN) has become more and more popular.WSN nodesare powered by batteries. In order to extend battery life, the power consumption of WSN receivers should be reduced. At the same time, the other performance of the receivers should be remained unchanged. As the first stage of the receivers, the LNAs greatly impact on the performance of the entire receivers. ${ }^{[1-2]}$

Two types of CMOS LNA topologies have become mainstream circuit implementations: common-source LNAs(CS-LNAs) and common-gate LNAs(CG-LNAs).In recent years, CG-LNAs have gained popularity because the

*Project supported by the National High Technology Research and Development Program (No. 2007AA01Z2A7) and the Special Fund of Jiangsu Province for the Transformation of Scientific and Technological Achievements (No. BA2010073). 
input impedance is fully or partially resistive. As a result, the impedance matching can be easily accomplished. Additionally, the impedance matching bandwidth of CG-LNAs is wider than that of CS-LNAs due to the resistive input impedance. Nevertheless, CS-LNAs have the best noise performance. Efforts have been made in the last few years to improve the noise performance of CG-LNAs. Solutions include the transformer-coupled and capacitor cross-coupled. ${ }^{[3-5]}$

\section{Circuits Structure}

The schematic of the proposed LNA is shown in Fig.1. Three pairs of MOSFETs adopt capacitorcross-coupled, which increases the effective $G_{\mathrm{m}}$ and decrease noise and power consumption. $\mathrm{M}_{1,2}$ and $\mathrm{M}_{3,4}$ are amplifying MOSFETs. The differential signal is inputtedto the source of $M_{1,2}$ and the gate of $M_{5,6} . M_{1}$ and $\mathrm{M}_{5}, \mathrm{M}_{2}$ and $\mathrm{M}_{6}$ adopt current-reuse structure to decrease power consumption.

\subsection{Capacitor cross-coupled}

Effective $\mathrm{G}_{\mathrm{m}}$ is increasedby adopting capacitorcross-coupledtechnique. The schematic of the boosting effective $G_{m}$ is shown in Fig.2(a). The structure of the capacitor cross-coupled CG-LNA is shown in Fig.2 (b).

As shown in Fig.2(a), an inverting amplifier is set between the gate and the source of the MOSFET. The inverting amplification value is A. Through the derivation, we can get the expression of the $\mathrm{NF}^{[5]}$ :

$$
F=1+\frac{\overline{i_{\mathrm{nd}}^{2}}}{e_{\mathrm{ns}}^{2}}\left(\frac{1}{(1+A) g_{\mathrm{m}} R_{\mathrm{s}}}\right)^{2}=1+\frac{\gamma g_{\mathrm{d} 0}}{(1+A)^{2} R_{\mathrm{s}} g_{\mathrm{m}}^{2}}=1+\left.\frac{\gamma}{\alpha(1+A)}\right|_{(1+A) g_{\mathrm{m}} R_{\mathrm{s}}=1}
$$

As shown in Fig.2(b), we can deduce ${ }^{[5]}$ :

$$
A=\frac{C_{\mathrm{C}}}{C_{\mathrm{C}}+C_{\mathrm{gs}}}=\frac{1}{1+C_{\mathrm{C}} / C_{\mathrm{gs}}} \approx 1
$$

So the NF of the LNA which adopts capacitor cross-coupled is ${ }^{[5]}$ :

$$
F=1+\frac{\gamma}{2 \alpha}
$$




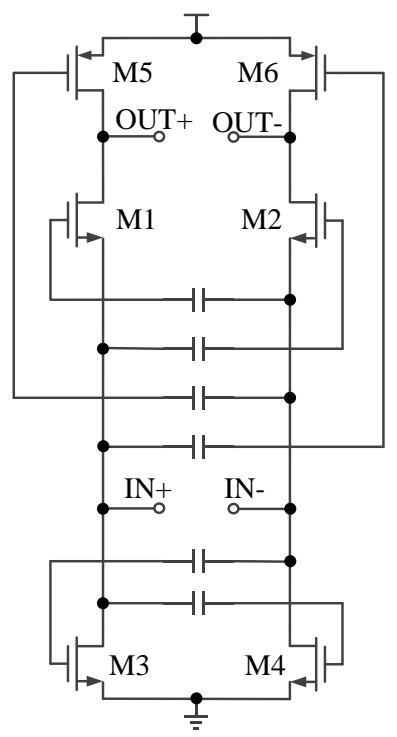

Fig.1Schematic of the proposed LNA

It can be seen that the second part of the equation is decreased by half. Meanwhile, capacitor cross-coupled can reduce power consumption. The reason is given in the next section.
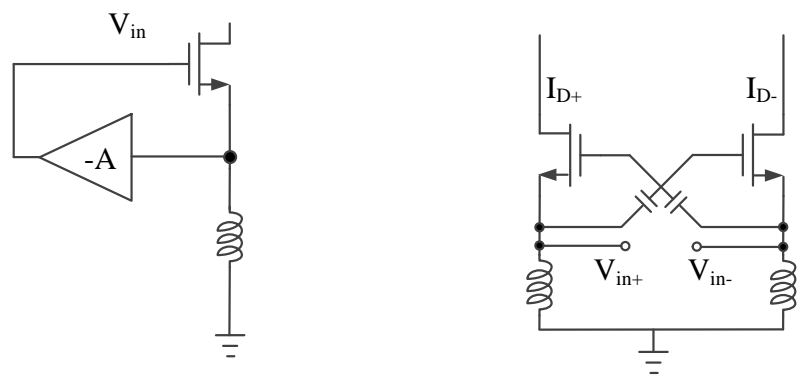

(a)(b)

Fig.2(a)schematic of the boosting effective $G_{m}$ (b)structure of the capacitor cross-coupled CG-LNA

\subsection{Impedance matching}

Fig. 3 shows the small signal representation of the proposed LNA. 


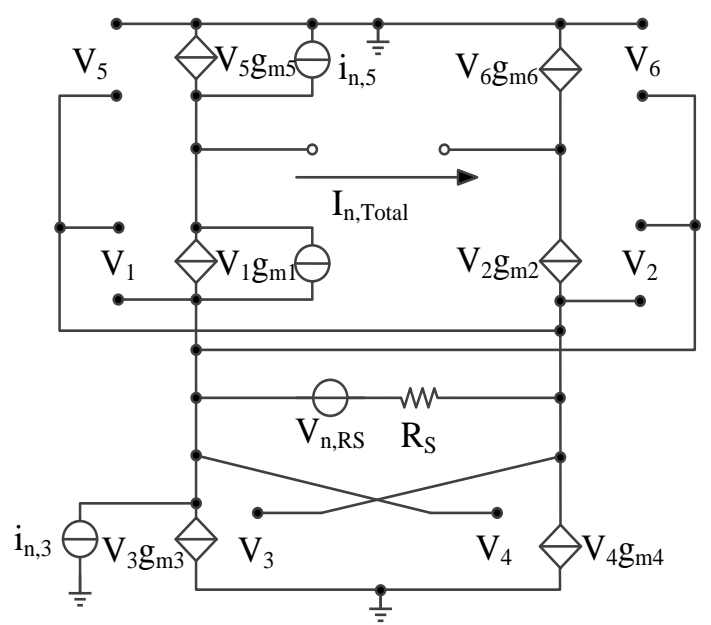

Fig.3small signal representation of the LNA

Ignoring $\mathrm{C}_{\mathrm{gs}}$ of the MOSFETs, the input impedance can be expressed as:

$$
Z_{\mathrm{in}}=\frac{1}{2 g_{\mathrm{m} 1}} / / \frac{-1}{g_{\mathrm{m} 3}}=\frac{1}{2 g_{\mathrm{m} 1}-g_{\mathrm{m} 3}}
$$

But this formulation only expresses the real part of the input impedance.There exists pathways through the capacitance to the ground. So the imaginary part of the input impedanceis large. By adjusting the capacitance and the $\mathrm{W}$ or L of the MOSFETs, we can decrease the imaginary part to accomplish impedance matching.

If the capacitor cross-coupled were not adopted, the input impedance is:

$$
Z_{\mathrm{in}}=\frac{1}{g_{\mathrm{m} 1}} / / \frac{-1}{g_{\mathrm{m} 3}}=\frac{1}{g_{\mathrm{m} 1}-g_{\mathrm{m} 3}}
$$

It can be seen that for the same $\mathrm{Z}_{\mathrm{in}}$, capacitor cross-coupled decreases $\mathrm{g}_{\mathrm{m} 1}$.Socurrentconsumption is also reduced. This is the reason that capacitor cross-coupled can reduce the power consumption.

\subsection{Noise analysis}

The main noise of MOSFET is the drain noise. So we only consider the drain noise, neglecting the other noise.

When the source is matched, the total transconductance is ${ }^{[4]}$ :

$$
G=\frac{1}{2}\left(g_{\mathrm{m} 1}+g_{\mathrm{m} 5} / 2\right)
$$


So the output noise current due to the source resistor is ${ }^{[4]}$ :

$$
i_{\mathrm{n}, \mathrm{Rs}}=\frac{1}{2} v_{\mathrm{n}, \mathrm{RS}}\left(g_{\mathrm{m} 1}+g_{\mathrm{m} 5} / 2\right)
$$

The noise contribution due to the $\mathrm{M}_{1,2}$ comes from two mechanisms that add to the output.

The first mechanism is due to part of $i_{n 1}$ entering into $M_{1,2}$ and producing an output current equal to ${ }^{[4]}$ :

$$
i_{\mathrm{n} 1, \mathrm{~d}}=i_{\mathrm{n} 1}\left(\frac{g_{\mathrm{m} 1}}{2\left(2 g_{\mathrm{m} 1}-g_{\mathrm{m} 3}\right)}-\frac{1}{2}\right)=i_{\mathrm{n} 1}\left(\frac{g_{\mathrm{m} 1} R_{\mathrm{s}}}{4}-\frac{1}{2}\right)
$$

The second one is due to part of $i_{\mathrm{n} 1}$ flowing through $R_{\mathrm{s}}$ and producing a voltage $\mathrm{V}_{43}$ that is converted into a differential output current. We can get a voltage $\mathrm{V}_{43}$ by using a Thevenin equivalent circuit ${ }^{[4]}$ :

$$
V_{43}=\frac{i_{\mathrm{n}, 1}}{2\left(2 g_{\mathrm{m} 1}-g_{\mathrm{m} 2}\right)}=\frac{i_{\mathrm{n}, 1} R_{\mathrm{s}}}{4}
$$

So the total noise current due to $\mathrm{M}_{1,2}$ is:

$$
i_{\mathrm{n} 1, \mathrm{~d}}=i_{\mathrm{n} 1}\left(\frac{g_{\mathrm{ml}} R_{\mathrm{s}}}{4}-\frac{1}{2}\right)+i_{\mathrm{n} 1} \frac{g_{\mathrm{m} 5} R_{\mathrm{S}}}{8}=\frac{i_{\mathrm{n} 1}}{2}\left(\frac{R_{\mathrm{S}}}{2}\left(g_{\mathrm{ml}}+\frac{g_{\mathrm{m} 5}}{2}\right)-1\right)
$$

When the equation (11) is satisfied, the noise of the input $\mathrm{M}_{1,2}$ can be cancelled. ${ }^{[4]}$

$$
g_{\mathrm{m} 5}=2\left(\frac{2-g_{\mathrm{m} 1} R_{S}}{R_{S}}\right)
$$

The differential noise current from $\mathrm{M}_{3,4}$ is ${ }^{[4]}$ :

$$
i_{\mathrm{n} 3 \mathrm{~d}}=i_{\mathrm{n} 3} \frac{g_{\mathrm{m} 1} R_{S}}{4}+i_{\mathrm{n} 3} \frac{g_{\mathrm{m} 5} R_{\mathrm{S}}}{8}=i_{\mathrm{n} 3} \frac{R_{\mathrm{S}}}{4}\left(g_{\mathrm{m} 1}+\frac{g_{\mathrm{m} 5}}{2}\right)
$$

The noise current from $\mathrm{M}_{5,6}$ is ${ }^{[4]}$ :

$$
i_{\mathrm{n} 5, \mathrm{~d}}=i_{\mathrm{n}, 5} / 2
$$

So the total NF of the LNA is ${ }^{[4]}$ :

$$
F_{\mathrm{LNA}}=1+\frac{\gamma g_{\mathrm{m} 1}\left(R_{\mathrm{S}} G-1\right)^{2}}{2 R_{\mathrm{S}} G^{2}}+\gamma\left(g_{\mathrm{m} 1} R_{\mathrm{S}}-1\right)+\frac{\gamma g_{\mathrm{m} 5}}{2 R_{\mathrm{S}} G^{2}}
$$

Where $\gamma$ is the MOSFET thermal noise coefficient. When (11) is applied, the NF of the LNA becomes ${ }^{[4]}$ : 


$$
F_{L N A}=1+\gamma\left(g_{m 1} R_{S}-1\right)+\gamma\left(2-g_{m 1} R_{S}\right)=1+\gamma
$$

\section{The simulation results}

This paper presents a low voltage low noise amplifier for $700 \sim 900 \mathrm{MHz}$ WSN applications in $0.18 \mu \mathrm{m}$ RF CMOS process.It consumes about $900 \mu \mathrm{A}$ current from $1 \mathrm{~V}$ power supply.The layout of the LNA is shown inFig. 4. All the post-simulation is done with a buffer. The post-simulation result of NF is shown in Fig.5. The NF equals to $3.0 \mathrm{~dB}$ at $800 \mathrm{MHz}$. The post-simulation of $\mathrm{S} 11$ is shown in Fig.6. During700 900MHz, S11 is -12.82 -12.7dB; Fig.7 shows the post-simulation of S21. During700 900MHz, S21 is $11.88 \sim 14 \mathrm{~dB}$; The post-simulation of S22 is shown in Fig.8. During 700 900MHz, S22 is $-16.52 \sim 16.59 \mathrm{~dB}$. At low gain mode, $\mathrm{IP}_{1 \mathrm{~dB}}$ is $-15 \mathrm{dBm}$ (as shown in Fig.9), and the IIP3 is $1 \mathrm{dBm}$ (as shown in Fig.10).The load of the LNA is down-conversion mixer. We set the input impedance of down-conversion mixer as $2 \mathrm{~K}$ ohm. The voltage gain is $22 \mathrm{~dB}$.The post-simulation results are concluded in TableI.

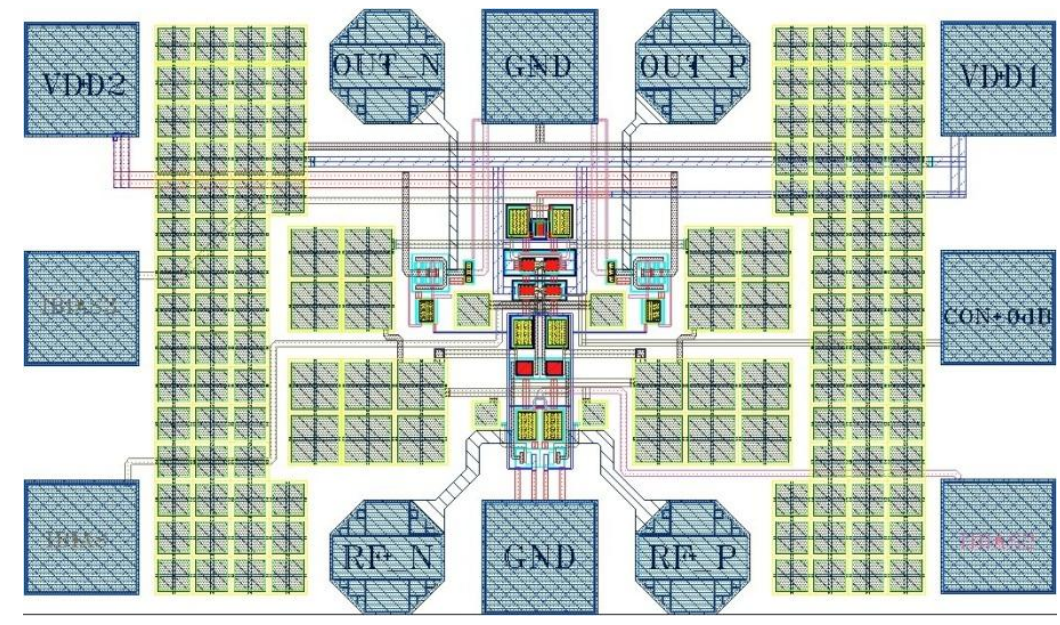

Fig. 4 Layout of the LNA 

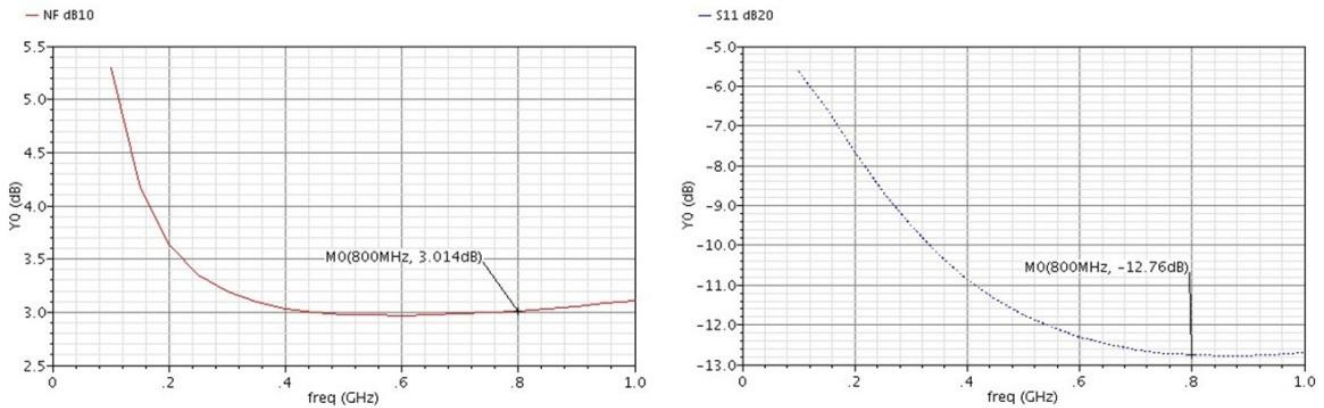

Fig.5Post-simulation result of NFFig.6Post-simulation result of S11
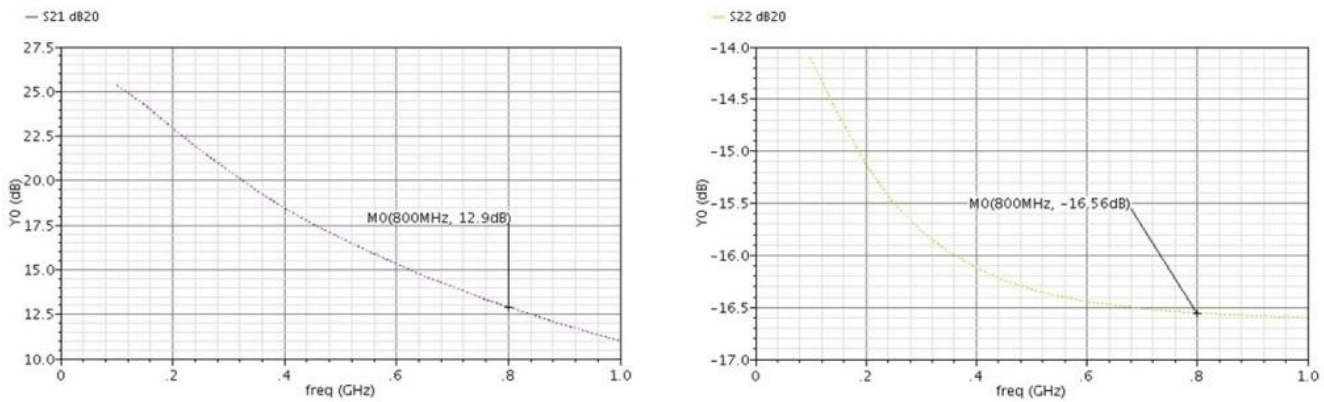

Fig.7Post-simulation result of S21Fig.8Post-simulation of S22
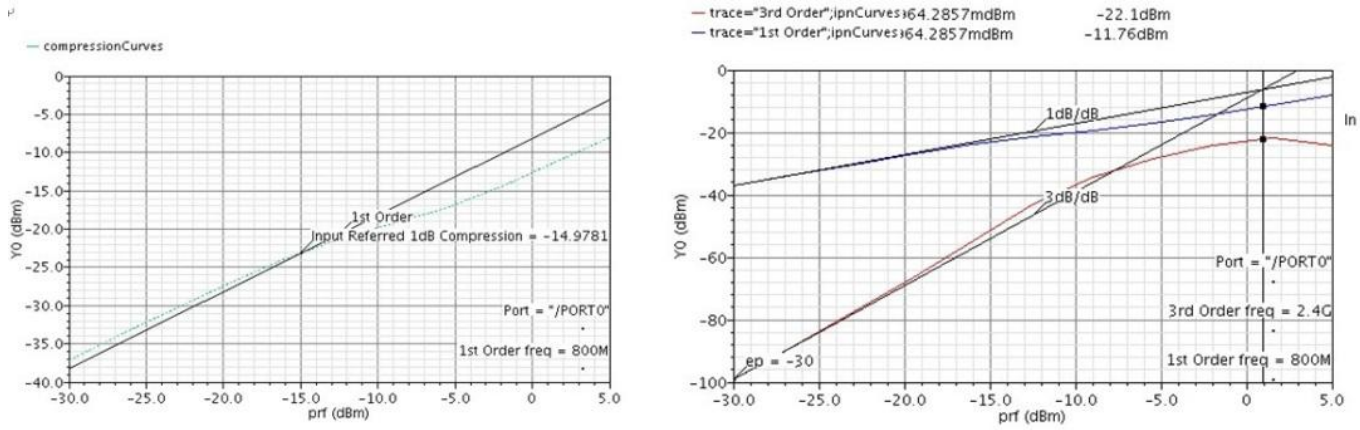

Fig.9Post-simulation of $\mathrm{IP}_{1 \mathrm{~dB}}$ Fig.10Post-simulation result of IIP3 
Table I.Summary of the LNA post-simulation results

\begin{tabular}{ll}
\hline Parameter & value \\
\hline Supply voltage $(\mathrm{V})$ & 1 \\
Power consumption $(\mathrm{mW})$ & 0.9 \\
$\mathrm{~S} 11(\mathrm{~dB})$ & $-12.82 \sim-12.7$ \\
$\mathrm{~S} 21(\mathrm{~dB})$ & $11.88 \sim 14$ \\
$\mathrm{~S} 12(\mathrm{~dB})$ & $-55.12 \sim-53.64$ \\
$\mathrm{NF}$ at high gain mode $(\mathrm{dB})$ & 2.82 \\
$\mathrm{IP}$ & -15 \\
$\mathrm{IIP}_{\mathrm{dB}}$ at low gain mode $(\mathrm{dBm})$ & -15 \\
\hline
\end{tabular}

\section{Summary}

This paper presents a low noise amplifier for $700 \sim 900 \mathrm{MHz}$ WSN applications in $0.18 \mu \mathrm{m}$ RF CMOS process. The power supply is $1 \mathrm{~V}$. The LNA adopts capacity cross-coupled, current-reuse and noise cancellation to decrease the NF and power consumption. Post-simulation results show that LNA can be fully adapted to WSN system applications.

\section{References}

[1] Kluge W, Poegel F, Roller H, et al. A fully integrated 2.4-GHz IEEE 802.15.4-compliant transceiver for ZigBee applications, IEEE J Solid-State Circuits, 2006, 41(12):2767

[2] Camus M, Butaye B, Garcia L, et al. A $5.4 \mathrm{~mW} / 0.07 \mathrm{~mm} 22.4 \mathrm{GHz}$ front-end receiver in 90 nm CMOS for IEEE 802.15.4 WPAN standard. IEEE JSSC, 2008, 43(6): 1372

[3] Zhang Hao, Li Zhiqun, Zhang Meng, et al. A $2.4 \mathrm{GHz}$ low-IF RF frontend for wireless sensor networks. Microwave and Millimeter Wave Technology (ICMMT), 2010: 225

[4] S. Rodriguez, A. Rusu ICT, A 6.4mW 1-3.5GHz Current-Mode Receiver Front-End with Noise Cancellation. IEEE978-1-4577-0704-9/102011.

[5] W. Zhuo, X. Li, S. Shekhar,et al, A Capacitor Cross-Coupled Common-GateLow-Noise Amplifier, IEEE TRANSACTIONS ON CIRCUITS AND SYSTEMS—II: EXPRESS BRIEFS, VOL. 52, NO. 12, DECEMBER 2005

[6] P.I. Mak and R. Martins, "Design of an ESD-protected ultra-wideband LNA in nanoscale CMOS for full-band mobile TV tuners,” IEEE Trans. Circuits Syst. I, vol. 56, May. 2009, p. 933-942. 